\title{
Instantons in asymptotically safe and free quantum field theories
}

\author{
Francesco Sannino, ${ }^{1, *}$ and Vedran Skrinjar ${ }^{2, \dagger}$ \\ ${ }^{1} \mathrm{CP}^{3}$-Origins \& the Danish Institute for Advanced Study, Campusvej 55, 5230 Odense, Denmark \\ ${ }^{2}$ International School for Advanced Studies, Via Bonomea 265, 34136 Trieste, Italy
}

(Received 6 August 2018; published 26 April 2019)

\begin{abstract}
We investigate the instanton dynamics of asymptotically safe and free quantum field theories featuring, respectively, controllable ultraviolet and infrared fixed points. We start by briefly reviewing the salient points about the instanton calculus for pure Yang-Mills (YM) and QCD theory. We then move on to determine the role of instantons within the controllable regime of the QCD conformal window. In this region we add a fermion-mass operator and determine the density of instantons per unit volume as a function of the fermion mass. Finally, for the first time, we extend the instanton calculus to asymptotically safe theories.
\end{abstract}

DOI: 10.1103/PhysRevD.99.085010

\section{INTRODUCTION}

The standard model and its four-dimensional extensions are described by gauge-Yukawa theories; it is therefore paramount to understand their dynamics.

Of special interest are theories that are fundamental according to Wilson [1,2], meaning that they are well defined at arbitrarily short distances. Asymptotically free [3,4] and safe [5] quantum field theories (QFT) are two classes of fundamental quantum field theories. For the former, at extremely short distances, all interactions vanish, while for the latter the interactions freeze. In theories with multiple couplings, some can be free and others can be safe. Although asymptotic freedom has a long and successful history, the discovery of four-dimensional controllable asymptotically safe quantum field theories is recent $[5,6]$. This result has enabled novel dark and bright extensions of the standard model [7-13].

The infrared dynamics of fundamental field theories is extremely rich, and it can entail confinement and/or chiral symmetry breaking or large distance conformality. This depends on the field content of the specific quantum field theory as well as the presence and type of infrared relevant operators such as scalar and fermion masses. In particular, asymptotically free theories can develop an interacting infrared (IR) fixed point that in certain limits is perturbatively controllable, known as the Banks-Zaks (BZ) [14]

\footnotetext{
*sannino@cp3.sdu.dk

†vskrin@sissa.it
}

Published by the American Physical Society under the terms of the Creative Commons Attribution 4.0 International license. Further distribution of this work must maintain attribution to the author(s) and the published article's title, journal citation, and DOI. Funded by SCOAP. fixed point. The full region in color-flavor space, for gauged fermion theories, where an IR fixed point is present, is known as the conformal window; see [15] for an introduction and [16] for a summary of recent lattice efforts. Recently, building on the large $N_{f}$ results of [17-21], the concept of the conformal window has been extended to include the asymptotically safe region at a large number of flavors for which asymptotic freedom is lost [22].

The first systematic study of exact constraints that a supersymmetric asymptotically safe quantum field theory must abide by, including $a$ maximization [23] and collider bounds [24], appeared in [25] extending the results of [26]. Here it was also established that Seiberg's SQCD conformal window [27] does not admit an asymptotically safe conformal region. This result is in net contrast with the nonsupersymmetric case [22]. Building upon the results of [25] in Ref. [28], the first evidence for supersymmetric safety was uncovered within the important class of grand unified theories. The generalization to different types of supersymmetric quantum field theories passing all known constraints appeared in [29].

Here we are concerned with generalizing and applying the instanton calculus to gauge theories in the perturbative regime of the QCD conformal window as well as of controllable nonsupersymmetric asymptotically safe quantum field theories $[5,6]$.

To keep the work self-contained, we briefly review the instanton calculus for pure Yang-Mills (YM) as well as QCD theory including its large- $N_{c}$ limit in Sec. II. Instantons for the QCD conformal window are introduced and discussed in Sec. III. Here, we consider the 2-loop corrected instantons that allow us to follow the perturbative renormalisation group (RG) flow deep in the infrared where a perturbative interacting IR fixed point occurs. We then perform our analysis in the fermion-mass deformed theory 
and derive the main instanton features as a function of the fermion mass operator. For example, we compute the density of instantons per unit volume as a function of the fermion mass measured in units of the RG invariant scale. The latter separates the infrared interacting theory from the UV free fixed point. Finally, we generalize the instanton calculus to safe rather than free theories in Sec. IV. Here, we consider again the fermion mass dependence that now, however, affects the infrared trivial fixed point. We offer our conclusions in Sec. V.

\section{INSTANTON CALCULUS REVIEW}

In QFT one aims at computing the partition function,

$$
Z[\mathcal{J}]=\int \mathcal{D} \phi e^{\mathrm{i} S[\phi ; \lambda]+\mathcal{J} \phi},
$$

where $S[\phi ; \lambda]$ is the sum of a classical action, a gauge-fixing action, and a ghost action, depending on the fields $\phi$ and the couplings $\lambda$, and $\mathcal{J}$ is a source for $\phi$. If the action is nonintegrable, one usually attempts to solve the problem through perturbation theory, which amounts to expanding the action in powers of small coupling constants $\lambda$. Solutions of the classical theory corresponding to $S[\phi ; \lambda]$ are specific classical field configurations $\bar{\phi}$. Since the first variation of the action vanishes on these configurations, they represent stationary points, or extrema, of the action. The integrand on the right-hand side (rhs) of (1) is clearly an oscillating function, and thus one may attempt to evaluate the integral by performing an expansion around the classical solution $\bar{\phi}$. Symbolically, we have

$$
Z[\mathcal{J}]=\int \mathcal{D} \phi e^{\mathrm{i}\left[S[\bar{\phi}]+\frac{1}{2} \phi S^{(2)}[\bar{\phi}] \phi+\mathcal{O}\left(\phi^{3}\right)\right]+\mathcal{J} \phi} .
$$

This is the core of the steepest descent method for addressing the issue of oscillating integrals. One defines the vacuum solution as the classical configuration that minimizes the energy functional (the Hamiltonian). In the case of (comparatively) simple QFTs, there is just one vacuum state, and thus there is but a single field configuration $\bar{\phi}$ around which one should expand the partition function. This is precisely the situation described by Eq. (2). For YM theories, often coupled to scalars or fermions, and occasionally coupled to gravity, the vacuum structure is more involved, and if one would naively apply the above prescription, several important phenomena would be unaccounted for, such as a deeper understanding of chiral symmetry breaking, the generation of the eta prime mass in QCD, etc.

Let us therefore reconsider briefly the correct approach applicable to a generic QFT [30-34]. We begin by Euclideanizing the QFT by performing the Wick rotation $t \rightarrow \tau=-\mathrm{i} t$. One should treat gauge fields and fermions with care during this procedure. Euclidean action $S_{E}$ is a functional of Euclidean fields $\phi_{E}(x)$ living on a 4D Euclidean space described by coordinates $x=\left(x_{1}, x_{2}\right.$, $\left.x_{3}, \tau\right)$. When solving the equations of motion, one has to set up the boundary conditions for $|x| \rightarrow \infty$ such that the action remains finite. Usually, our conditions require $\phi \rightarrow$ const for $|x| \rightarrow \infty$. If the potential has only one extremum, there is a single vacuum solution (constant field configuration in all of the space), and therefore the naive perturbation theory described by (2) is valid. If, however, the potential has more than one degenerate vacuum, then there exist classical solutions interpolating between these Euclidean vacua. These finite-action topologically stable solutions to classical Euclidean equations of motion are called instantons or pseudoparticles [35,36]. Instantons are topologically stable since going from one such field configuration to another would require bridging an infinite action barrier.

It is now clear that the correct application of the steepest descent method to the Euclideanized version of (2) involves a summation over all instanton configurations. Even though one does not find instantons as classical solutions to Lorentzian equations of motion, it is clear that the Lorentzian partition function can be obtained by Wick rotating the Euclidean partition function, and thus instantons have to be incorporated in the Lorentzian computation. Being interpreted as fields that interpolate between different vacua, instantons are crucial for understanding the rich vacuum structure in YM theories.

When discussing instantons, the $S U(2)$ color group plays a special role since $S U(N)$ instantons can be determined starting from the $S U(2)$ case $[30,37]$. Let us therefore assume for the moment that we have a Euclidean YM action,

$$
S[A]=\frac{1}{4} \int_{x} G_{a}^{\mu \nu}(A) G_{a \mu \nu}(A)
$$

where $\int_{x} \equiv \int d^{4} x \equiv \int d^{3} x d \tau$, and $A_{\mu}^{a}$ is the gauge field. To find instanton solutions we require the action to be bounded, but rather than asking that $A_{\mu}^{a}(x)$ decays faster than $1 / x$ for $|x| \rightarrow \infty$, we require it to become pure gauge,

$$
A_{\mu} \stackrel{|x| \rightarrow \infty}{\longrightarrow} \mathrm{i} S \partial_{\mu} S^{\dagger},
$$

where $S$ are $S U(2)$ matrices (not to be confused with the action) that depend on angles only. Here, $S U(2)$ instantons are related to maps from $S U(2)$ to itself. Such maps are classified by the third homotopy group, and they fall into topologically distinct classes. In the case of $S U(2)$, these are labeled by integer numbers, and members from different classes cannot be continuously mapped into each other. Instantons belonging to the same class are related by a gauge transformation.

The integers labeling distinct topological classes of instantons can be thought of as topological charges. 
Furthermore, for a given instanton configuration, the topological charge is given by

$$
n=\frac{g^{2}}{32 \pi^{2}} \int_{x} G_{a}^{\mu \nu} \tilde{G}_{a \mu \nu}, \quad n \in \mathbb{Z},
$$

where $g$ is the gauge coupling. One can complete the square in the action as follows (suppressing indices),

$$
\begin{aligned}
S & =\frac{1}{4} \int_{x} G G=\int_{x} \frac{1}{4} G \tilde{G}+\frac{1}{8}(G-\tilde{G})^{2} \\
& =n \frac{8 \pi^{2}}{g^{2}}+\frac{1}{8} \int_{x}(G-\tilde{G})^{2} .
\end{aligned}
$$

For the instanton with topological charge $n$, the action clearly takes the following minimum value:

$$
\left.S\right|_{n \text {-instanton }}=n \frac{8 \pi^{2}}{g^{2}}
$$

[Strictly speaking, Eq. (8) holds for positive $n$. Negative values of $n$ are obtained via a parity transformation since then $G \tilde{G} \rightarrow-G \tilde{G}$. Following the same argument as above, the action reaches its minimum at $|n| \frac{8 \pi^{2}}{g^{2}}$ for the field configuration, which is anti-self-dual, $G=-\tilde{G}$. Such a field configuration is called an anti-instanton.] This is achieved when the field satisfies the self-duality condition, $G=\tilde{G}$. Using Bianchi identities, one can show that the field satisfying the self-duality condition is on shell; i.e., it automatically satisfies the equations of motion. Computing the value of the action on an instanton solution constitutes the first important result of the instanton calculus. Starting from the asymptotics, Eq. (4), and assuming the same directional dependence of the solution in all spacetime points, one can write an ansatz for the instanton. Requiring the absence of singularities at the origin of space and selfduality of the solution suffices to uniquely fix the instanton (up to collective coordinates) [30]. This result is the famous Belavin-Polyakov-Schwarz-Tyupkin (BPST) instatons [SU(2) instanton with charge $n=1$ ] [35]. Explicitly,

$$
A_{\mu}^{a}=\frac{2}{g} \eta_{a \mu \nu} \frac{\left(x-x_{0}\right)^{\nu}}{\left(x-x_{0}\right)^{2}+\rho^{2}} .
$$

The above expression for the BPST instanton is in the socalled regular gauge. The parameter $\rho$, the instanton size, is the aforementioned integration constant, and it is one of the instanton collective coordinates. The remaining collective coordinates are the instanton position in spacetime, $x_{0}$, and its orientation in color space. Finally, $\eta_{a \mu \nu}$ are known as 't Hooft symbols [36].

The generalization to simple Lie algebras is obtained directly from the $S U(2)$ BPST instanton, exploiting the fact that any $S U(N)$ group contains $S U(2)$ subgroups.
To deduce the $S U(N)$ instantons, one simply embeds the BPST solution (9) into $S U(N)$. This choice of embedding is ambiguous, but the most common choice is the so-called minimal embedding. It consists in taking the $S U(N)$ generators in the fundamental group and taking the first three generators $T^{1}, \ldots, T^{3}$ to be block-diagonal, with $S U$ (2) generators embedded in the upper-left corner. The $S U(N)$ BPST instanton is obtained by contracting the first three generators $T^{a}, a=1,2,3$, with the BPST solution (9). One can analogously obtain $S U(N)$ instantons with charge $n \neq 1$ from other $S U(2)$ solutions. This simple prescription works because the third homotopy group of $S U(N)$ is $\mathbb{Z}$ for all $N$, and with the minimal embedding, each equivalence class of $S U(N)$ solutions contains a representative $S U(2)$ instanton.

\section{A. The QCD story}

Here, we show that an instanton ensemble plays an important role in determining the structure of the QCD vacuum. We start by reviewing the construction of a partition function for such an ensemble. We begin with the famous result for the one-instanton partition function, which was given by 't Hooft in 1976 [36].

The vacuum-to-vacuum transition amplitude in the presence of a single instanton is given by the following 1-loop instanton calculus result for an $S U\left(N_{c}\right)$ pure YangMills theory [36,38],

$$
\begin{aligned}
W^{(1)}= & \frac{4}{\pi^{2}} \frac{\exp \left(-\alpha(1)-2\left(N_{c}-2\right) \alpha(1 / 2)\right)}{\left(N_{c}-1\right) !\left(N_{c}-2\right) !} \\
& \times \int d^{4} x d \rho \rho^{-5}\left(\frac{4 \pi^{2}}{g_{0}^{2}}\right)^{2 N_{c}} \exp \left(-\frac{8 \pi^{2}}{g_{1 L}^{2}}\right) \\
\equiv & C_{c} \int d^{4} x d \rho \rho^{-5}\left(\frac{8 \pi^{2}}{g_{0}^{2}}\right)^{2 N_{c}} \exp \left(-\frac{8 \pi^{2}}{g_{1 L}^{2}}\right) .
\end{aligned}
$$

The integral on the rhs is over the instanton size $\rho$, and its integrand is referred to as the instanton density. Note that the numerical factor $C_{c}$ depends only on the number of colors, and it also contains the factor $2^{-2 N_{c}}$. The above integral is IR divergent $[\rho \rightarrow \infty$, see (15)] because of the running coupling in the exponent. Clearly one has to tame this behavior for the result to be meaningful.

If the Yang-Mills theory is coupled to $N_{f}$ Dirac fermions, then, at 1 loop, they contribute via the fermion determinant to the above result. It is both possible and useful to separate the zero and nonzero fermionic modes. The nonzero modes contribute to the exponential as [36]

$$
\exp \left[-\frac{2 N_{f}}{3} \log \left(\rho / \rho_{0}\right)+2 N_{f} \alpha(1 / 2)\right],
$$

where the first term is the fermion contribution to the 1-loop running of the gauge coupling and $\alpha(x)$ is a function 
defined in [36]. ${ }^{1}$ Taking all the fermions to have the same mass $m$, the zero modes contribute a term

$$
(m \rho)^{N_{f}} .
$$

We can now generalize the result in (11) to include fermions using the 1-loop running of the QCD gauge coupling,

$\frac{8 \pi^{2}}{g_{1 L}^{2}}=\frac{8 \pi^{2}}{g_{0}^{2}}-b \log \left(\rho / \rho_{0}\right), \quad b=\frac{11}{3} N_{c}-\frac{2}{3} N_{f}$,

and derive

$$
\begin{aligned}
W^{(1 L)}= & \frac{4}{\pi^{2}} \frac{\exp (-\alpha(1)+4 \alpha(1 / 2))}{\left(N_{c}-1\right) !\left(N_{c}-2\right) !} \\
& \times \exp \left(2\left(N_{f}-N_{c}\right) \alpha(1 / 2)\right) m^{N_{f}}\left(\frac{4 \pi^{2}}{g_{0}^{2}}\right)^{2 N_{c}} \\
& \times \int d^{4} x d \rho \rho^{-5+N_{f}} \\
& \times \exp \left(-\frac{8 \pi^{2}}{g_{0}^{2}}+\left(\frac{11}{3} N_{c}-\frac{2}{3} N_{f}\right) \log \left(\rho / \rho_{0}\right)\right) \\
= & C_{c f} m^{N_{f}} \int d^{4} x d \rho \rho^{-5+N_{f}}\left(\frac{8 \pi^{2}}{g_{0}^{2}}\right)^{2 N_{c}} \exp \left(-\frac{8 \pi^{2}}{g_{1 L}^{2}}\right) .
\end{aligned}
$$

Besides suffering from the divergence of the instanton density for large instantons, the master equation (15) has another important feature. The zero mode contributions of (13) imply the vanishing of the whole amplitude as $m \rightarrow 0$. This was noted and thoroughly discussed in [36], see also $[39,40]$.

A simple strategy to bypass this problem was initially given by [39]. Their reasoning goes as follows. It is empirically known that the QCD vacuum is a medium in which many condensates form, so instead of studying a single instanton in isolation, one should take into account the condensation phenomenon on the instanton density. In particular, the authors focused on the chiral condensate $\langle\bar{q} q\rangle$. At the time, one could not determine the chiral condensate from first principles, so the authors employed its phenomenological value. Besides its relevance as an order parameter for the spontaneous breakdown of chiral symmetry (SBCS), one considers the chiral condensate as a dynamical fermion mass that should be used in the amplitude (15) instead of the bare mass. Following [39], we compute the effective quark mass in the presence of a nonvanishing $\langle\bar{q} q\rangle$ condensate in QCD to be given by

\footnotetext{
${ }^{1} \alpha(1 / 2)=0.145873$ and $\alpha(1)=0.443307$.
}

$$
\begin{aligned}
m_{\mathrm{eff}} & =m-\frac{4 \pi^{2} \rho^{2}}{N_{c}}\left\langle\bar{q}^{L} q^{R}\right\rangle \\
& =m-\frac{2 \pi^{2} \rho^{2}}{N_{c}}\left\langle\bar{q}\left(1+\gamma_{5}\right) q\right\rangle .
\end{aligned}
$$

Crucially, in the case $m \rightarrow 0$, the effective mass does not vanish, meaning that the vacuum-to-vacuum transition amplitude in the presence of a single instanton is nonzero provided that the chiral condensate forms. In this way, the authors of [39] successfully pointed towards the physical mechanism responsible for resolving the issue with the zero mass limit.

Let us now return to the other issue, the IR divergences of the instanton density. Conceptually, it is reasonable to expect that if QCD forms gluon condensates, then they should be described by a statistical ensemble of the instantons forming them. The early attempts in this direction imagined the QCD vacuum to be described by an instanton gas [34,41]. This was demonstrated to be a poor description of the physical vacuum since instantons were much more strongly interacting. An important step forward in understanding the QCD vacuum came with Shuryak's phenomenological instanton liquid model in 1982 [42]. He showed that a simple ad hoc model of the instanton medium as a liquid with only two free parameters can describe a number of nuclear physics observables reasonably well. His model assumes that all instantons have the same size, $\bar{\rho}$, and he obtained the instanton size and the density of the instanton liquid from the empirical value of the gluon condensate. Thus, the approach does not explain why the instanton density is a deltalike peak around some $\bar{\rho}$, but such an ad-hoc phenomenological description has predictive power and it seems to explain nuclear physics data well.

The above ideas were developed more systematically within the mean field approximation by Diakonov and Petrov in 1983 and 1985, aiming at a description of an ensemble of instantons from the first principles. The failure of the instanton gas picture implied that the instanton interactions should be modeled even if the medium itself turns out to be rather dilute. This was motivated by the expectation that the instanton interactions would remove the IR divergence. Thus, the authors in [43] introduced a modified variational procedure in an attempt to approximate the exact multi-instanton partition function. They applied their method to pure Yang-Mills theory, and besides curing the IR problem, they also successfully computed a number of physical observables. In a later work [40], the method was extended to include gauged fermions. The central result of that paper is that, in an instanton background, fermions develop a momentum-dependent effective mass which is nonvanishing in the zero momentum limit, confirming the expectations of Ref. [39] as summarized above. 
Before moving on to the large- $N_{c}$ theory, we comment on one more issue regarding the master equation (16). It follows from the 1-loop computation that the coupling in the exponential term is renormalized, but the one in the preexponential factor is not. In the literature, this problem is often addressed by recognizing that at 2 loops the preexponential factor gets renormalized [43], and thus one replaces the bare coupling by the 1-loop running coupling and the 1-loop coupling by the 2-loop coupling. For completeness, we also provide the standard result for the 2-loop running coupling [39,44],

$$
\begin{aligned}
\frac{8 \pi^{2}}{g_{2 L}^{2}} & =\frac{8 \pi^{2}}{g_{0}^{2}}-b \log \frac{\rho}{\rho_{0}}+\frac{b^{\prime}}{b} \log \left(1-\frac{g_{0}^{2}}{8 \pi^{2}} \log \frac{\rho}{\rho_{0}}\right), \\
b^{\prime} & =\frac{51}{9} N_{c}^{2}-\frac{19}{3} N_{f} .
\end{aligned}
$$

Note that the behavior of the coupling given in (19) is not the exact 2-loop one. In fact, this is only the leading UV contribution valid in the deep UV regime for the asymptotically free phase of QCD. ${ }^{2}$ We elaborate more on this point in Sec. III.

\section{B. Large $N_{c}$}

Pure Yang-Mills theory at large $N_{c}$ is an important step towards studying instantons in the conformal window as well as asymptotically safe instantons. In fact, many of the formulas derived in this subsection can be adapted to include the effects of fermions in these theories. Herein, we briefly outline the variational approach of [43] and present their main results. We particularly focus on the large- $N_{c}$ limit following Ref. [45].

Assuming that the pure YM vacuum is given by a background gauge field configuration which consists of a large set of instantons, following [43], in the absence of exact results in pure YM theory, one approximates such a background to be a sum of simple, localized 1-instanton solutions. Starting from such an ansatz, the ground state can be derived by introducing a modification of the Feynman's variational principle. The modified variational principle is used to approximate a partition function $Z$,

$$
Z=\int \mathrm{D} \phi e^{-S[\phi]} .
$$

It consists in taking the action $S$, modifying it slightly to get an action $S_{1}$ so that $S_{1}$ has the minimum on the ansatz field configuration, and then using the inequality

$$
Z=\left(\frac{1}{Z_{1}} \int D \phi e^{-\left(S[\phi]-S_{1}[\phi]\right)} e^{-S_{1}[\phi]}\right) Z_{1} \geq Z_{1} e^{-\left\langle S-S_{1}\right\rangle}
$$

\footnotetext{
${ }^{2}$ This is clear since the expression (19) is manifestly ignorant of the possible existence of a perturbative IR fixed point.
}

where $Z$ is the partition function that we want to approximate using the variational principle. Note that $Z$ is given by (21) and $Z_{1}$ is defined analogously, with the action $S_{1}$. The expectation values $\langle$.$\rangle are taken with respect to the$ measure $\exp \left(-S_{1}\right)$.

Let us take the background field to be given by $\bar{A}=\sum_{I} A_{I}+\sum_{\bar{I}} A_{\bar{I}}$, where $I$ runs over the instanton configurations and $\bar{I}$ over anti-instantons. We may rewrite the Lagrangian as follows,

$$
\begin{aligned}
-\frac{1}{4 g^{2}} F^{2}(\bar{A}) & =-\frac{1}{4 g^{2}}\left(\sum_{i=I, \bar{I}} F^{2}\left(A_{i}\right)+F^{2}(\bar{A})-\sum_{i=I, \bar{I}} F^{2}\left(A_{i}\right)\right) \\
& \equiv-\frac{1}{4 g^{2}}\left(\sum_{i=I, \bar{I}} F^{2}\left(A_{i}\right)+U_{\mathrm{int}}\right)
\end{aligned}
$$

where the first term is the Lagrangian of a noninteracting instanton gas, and the second term describes the interaction in the medium. From here on, we use the notation $1 / 4 g^{2} F^{2}=1 / 4 G^{2}$. Including the bosonic statistics factors $N_{ \pm}$in front of the partition function, normalizing both sides of (22) to the perturbation theory vacuum, and regularizing the determinants, at 1-loop order we obtain the following expression,

$$
\begin{aligned}
\left.\frac{Z}{Z_{p t b}}\right|_{\mathrm{reg}, 1 L} & \geq \frac{1}{N_{+} ! N_{-} !} \int \prod_{i}^{N_{+}+N_{-}} d \gamma_{i} d\left(\rho_{i}\right) e^{-\beta(\bar{\rho}) U_{\mathrm{int}}\left(\gamma_{i}\right)} \\
& \equiv \frac{1}{N_{+} ! N_{-} !} \int \prod_{i}^{N_{+}+N_{-}} d \gamma_{i} e^{-E\left(\gamma_{i}\right)}
\end{aligned}
$$

In this expression $\gamma_{i}$ represents the collective coordinates of the $i$ th pseudoparticle (see Sec. II). Here, $d(\rho)$ stands for the 1-instanton density (11), and we use the standard notation

$$
\beta(\rho) \equiv 8 \pi^{2} / g^{2}(\rho)
$$

In the expression (24) $\beta(\rho)$ is renormalized by 1-loop determinants at a scale $\bar{\rho}$ corresponding to the average instanton size. In the second step of (24), we introduced the compact notation,

$$
E\left(\gamma_{i}\right)=\beta(\bar{\rho}) U_{\mathrm{int}}\left(\gamma_{i}\right)-\sum_{i} \log d\left(\rho_{i}\right) .
$$

If the medium is sufficiently dilute, one can consider only two-particle interactions in the interaction term, all the other ones being subdominant. ${ }^{3}$ This is the key physical ingredient beyond the simple instanton gas model. The interaction potential has been determined in [43].

\footnotetext{
${ }^{3}$ In fact, first corrections to this computation come not from considering higher order interactions but from considering 2-loop beta functions [43].
} 
Integrating over the relative angle between two instantons in color space, and integrating over the instanton separation, one obtains a remarkably simple expression,

$U_{\text {int }}^{2-\text { body }}\left(\rho_{1}, \rho_{2}\right)=\gamma^{2} \rho_{1}^{2} \rho_{2}^{2}, \quad \gamma^{2}=\frac{27 \pi^{2}}{4} \frac{N_{c}}{N_{c}^{2}-1}$,

where $\rho_{1,2}$ are the sizes of the two pseudoparticles, and the coupling $\gamma^{2}$ has the characteristic $1 / N_{c}$ behavior.

We may now use the variational principle. Assuming that the effect of the 2-body interactions can be well captured by a modification of the 1-instanton densities $d(\rho)$, we write

$$
E_{1}\left(\gamma_{i}\right)=-\sum_{I}^{N_{+}} \log \mu_{+}\left(\rho_{I}\right)-\sum_{\bar{I}}^{N_{-}} \log \mu_{-}\left(\rho_{\bar{I}}\right),
$$

where $\mu_{ \pm}$are effective densities to be determined through maximization of the rhs of (22). To explicitly write the rhs of (22) as a function of $\mu_{ \pm}$, we first need $Z_{1}$. This is obtained by substituting $E_{1}$ in place of $E$ in (24), which gives us

$$
Z_{1}=\frac{1}{N_{+} ! N_{-} !} V^{N_{+}+N_{-}}\left(\mu_{+}^{0}\right)^{N_{+}}\left(\mu_{-}^{0}\right)^{N_{-}},
$$

where

$$
\mu_{ \pm}^{0}=\int_{0}^{\infty} d \rho \mu_{ \pm}(\rho)
$$

The second piece we need to evaluate (22) is $\left\langle E-E_{1}\right\rangle$. We express it in terms of

$$
\overline{\rho_{ \pm}^{2}}=\frac{1}{\mu_{ \pm}^{0}} \int d \rho \rho^{2} \mu_{ \pm}(\rho) .
$$

Now we can substitute $Z_{1}$ and $\left\langle E-E_{1}\right\rangle$ in the rhs of (22) and minimize it with respect to (w.r.t.) $\mu_{ \pm}$. The following computation is not difficult, but it is a bit lengthy so we refer the reader to [43] for additional steps. There is an arbitrary constant appearing in the optimal $\mu_{ \pm}$, and if it is chosen to be equal, then $\mu_{+}=\mu_{-} \equiv \mu$. Writing $N_{+}+N_{-}=N$, we find the optimal $\mu$ to be

$$
\mu(\rho)=d(\rho) \exp \left(-\frac{\beta \gamma^{2} N}{V} \overline{\rho^{2}} \rho^{2}\right),
$$

where $\beta \equiv \beta(\bar{\rho})=8 \pi^{2} / g^{2}(\bar{\rho})$. This can be reinserted in (31) to give

$$
\left(\overline{\rho^{2}}\right)^{2}=\frac{\nu}{\beta \gamma^{2} N / V}, \quad \nu=\frac{b-4}{2} .
$$

This expression can be further inserted in the optimal $\mu$, and $\mu^{0}$ can be easily found using the explicit form of the optimal $\mu$ and of the 1-instanton density. Finally, we can determine the rhs of (29); see [43] for more details.

Instead of keeping the number of pseudoparticles $N$ fixed, we can work in the grand canonical ensemble. This allows us to find the average number of instantons in the medium by maximizing the rhs of (29) as a function of $N$. For the bosonic factors we set $N_{ \pm} !=(N / 2)$ ! and use the Stirling approximation. This brings us to the following important expression for the average instanton number:

$$
\langle N\rangle=V \Lambda_{\mathrm{YM}}^{4}\left(\Gamma(\nu) C_{c f} \tilde{\beta}^{2 N_{c}}\left(\beta \gamma^{2} \nu\right)^{-\nu / 2}\right)^{\frac{2}{\nu+2}},
$$

where $\tilde{\beta}=8 \pi^{2} / g_{0}^{2}$. Note that $\overline{\rho^{2}}$ enters this equation through $\beta=8 \pi^{2} / g_{1 L}^{2}\left(\overline{\rho^{2}}\right)$, so (33) and (34) should be solved simultaneously (consistently). The importance of the average number of instantons comes from the fact that it is related to the gluon condensate, vacuum energy, and topological susceptibility, and in a theory with fermions, to the $U(1)$ axial anomaly.

Substituting the optimal effective density $\mu$ in the rhs of (22), in terms of the number of instantons per unit volume, the partition function takes the following simple form:

$$
Z=\exp \left[\frac{1}{2}(\nu+1)\langle N\rangle\right]
$$

We can solve numerically for the expectation values of the instanton size and of the density of instantons in the vacuum. To do that we need to perform the aforementioned $\mathrm{RG}$ improvement by promoting $\tilde{\beta}$ to $\beta$ and $\beta$ to $8 \pi^{2} / g_{(2 L)}^{2}(\bar{\rho})$. Note that it is useful to introduce a free parameter $a$, called the fudge factor, in the log term of the 1-loop running coupling (14). The fudge factor essentially parametrizes the uncertainty on the actual confining scale $\Lambda_{\text {YM }}$. The numerical results are shown in Fig. 1. Even for modest values of $N_{c}$ shown in the figure, one already notices that the density of instantons increases as $\mathcal{O}\left(N_{c}\right)$, whereas the average instanton size is quite independent of $N_{c}$ and is always of $\mathcal{O}(1)$.

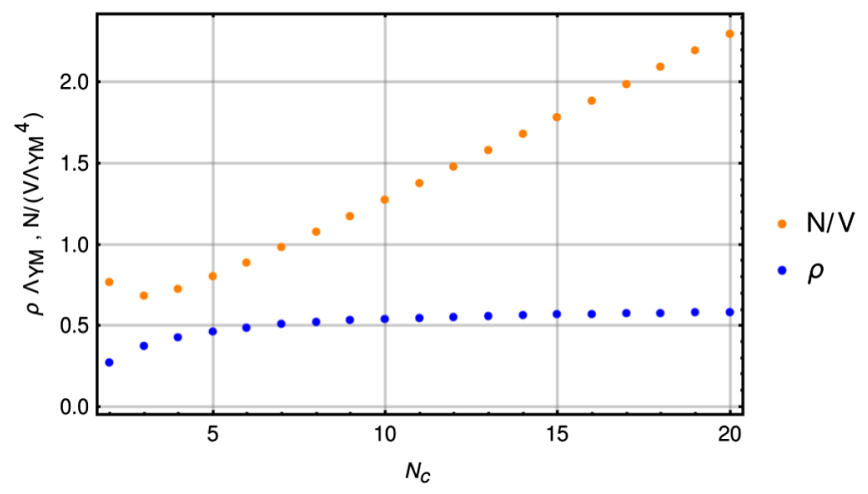

FIG. 1. Instanton size and density of instantons as a function of $N_{c}$. 


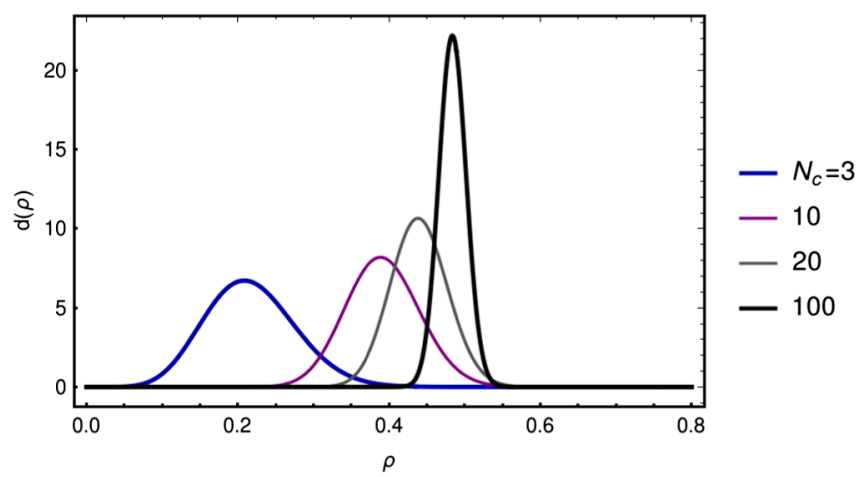

FIG. 2. Effective instanton density profile as a function of $N_{c}$ (normalized to unity).

We can also study the dependence of the effective instanton density $d(\rho)$ on the number of colors $N_{c}$. The results are shown in Fig. 2. Already from (10) we know that the amplitude decreases rapidly with $N_{c}$, but what we consider here is the shape and the spread of the distribution. (To this end we normalize all the distributions to $\mu^{0}=1$.) In particular, we notice that the distribution has a prominent peak centered about the average instanton size, and in the large $N_{c}$ limit, it becomes essentially deltalike [45].

Recall the relation between the full Lagrangian and the instanton gas Lagrangian, $F^{2}=\sum_{i} F_{i}^{2}+32 \pi^{2} U_{\text {int }}$. Since we know the value of the action for a BPST instanton [see Eq. (8)], we know that

$$
\left\langle\int \frac{d^{4} x}{32 \pi^{2}} F^{2}\right\rangle=\langle N\rangle+\left\langle U_{\text {int }}\right\rangle
$$

and from (24), it follows that $\left\langle U_{\text {int }}\right\rangle=-\partial \log Z / \partial \beta$. From (35) we thus obtain

$$
\left\langle U_{\text {int }}\right\rangle=\frac{\nu}{2 \beta}\langle N\rangle
$$

Figure 3 shows the ratio of the interaction energy to the free energy. Since the free energy is larger than the

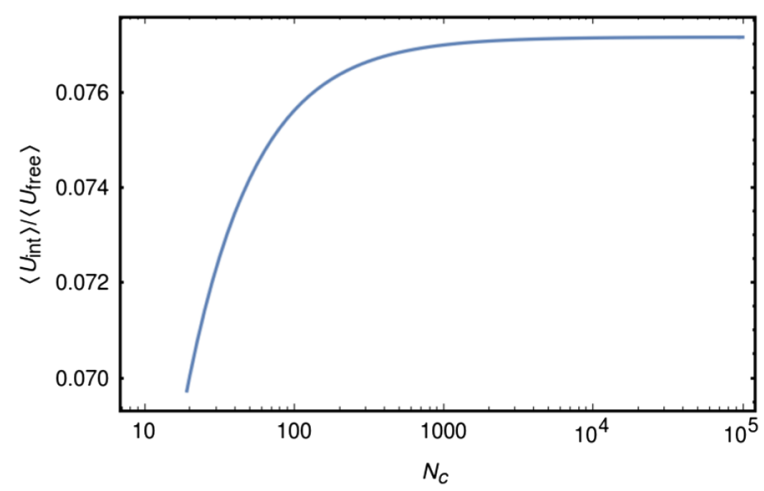

FIG. 3. Ratio of interaction energy to free energy as a function of $N_{c}$. We fixed $a=1 / 10$. interaction energy, we can trust the simplified 2-body interaction model. Further, because the gluon field VEV is related to the trace of the stress energy tensor (SET) by the scale anomaly relation, and since the trace of the SET is in direct relation to the vacuum energy density, we obtain the following leading-order expression for the vacuum energy density,

$$
\mathcal{E}=-\frac{b}{4} \frac{\langle N\rangle}{V}
$$

Notice that it grows quadratically with $N_{c}$, with an additional factor of $N_{c}$ with respect to noninteracting instanton gas [45].

Let us now compute the topological susceptibility. This is of particular interest because it is an observable. We start by adding the topological theta term, $\frac{i \theta}{32 \pi^{2}} \int d^{4} x F \tilde{F}$, to the action. The topological susceptibility is defined by

$$
\chi_{\text {top }}=-\left.\frac{\partial^{2} \log Z}{\partial \theta^{2}}\right|_{\theta=0}=\left\langle\left(\int d^{4} x \frac{F \tilde{F}}{32 \pi^{2}}\right)^{2}\right\rangle .
$$

In particular, adding the $\theta$-term to the partition function does not modify the computation of $\mu(\rho)$, or $\overline{\rho^{2}}$, and thus the only modification to (35) is an additional term $+i \theta\left(N_{+}-N_{-}\right)$. Self-consistently, by rewriting this as

$$
Z=\exp \left[\frac{\nu+2}{2}\langle N\rangle\left(1-\frac{\theta^{2}}{\nu+2}+\mathcal{O}\left(\theta^{4}\right)\right)\right],
$$

and taking the derivative as in (39), we get [43]

$$
\chi_{\text {top }}=\langle N\rangle .
$$

We are now ready to investigate and extend the role of instantons within the conformal window of QCD and for asymptotically safe quantum field theories.

\section{CONFORMAL WINDOW INSTANTONS}

In this section we determine the instanton dynamics in the QCD IR conformal window. We are prevalenty concerned with the calculable part of the conformal window, the one in which an IR fixed point is reached perturbatively, and it is often referred to as Banks-Zaks [14].

The perturbative IR fixed point occurs for the number of fermions $N_{f}$ tuned to be slightly below $11 / 2 N_{c}$ in the large- $N_{c}$, large- $N_{f}$ limit. In this limit one introduces an expansion in the physical parameter $\epsilon$, defined in (44), that measures the distance, in flavor space, from the loss of asymptotic freedom. This parameter can be made arbitrarily small. The fixed point value, being an expansion in $\epsilon$, can be made arbitrarily weakly interacting, rendering the expansion controllable. In Fig. 4 we compare the running 


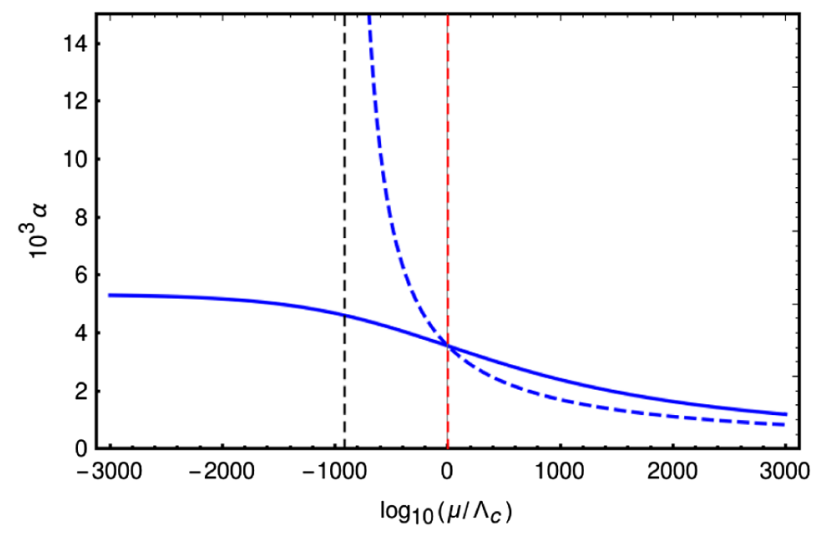

FIG. 4. Banks-Zaks running, shown as a continuous blue line for $\epsilon=-1 / 10$, interpolates between an interacting fixed point in the IR and a noninteracting fixed point in the UV. This result is obtained starting at 2 loops, whereas the analogous 1-loop running is given by the dashed blue line. The only scale in the BZ theory is the RG-invariant scale $\Lambda_{c}$ corresponding to the dashed red line. We choose the matching conditions such that the 1-loop and the 2-loop couplings match at the scale $\Lambda_{c}$; this fixes the 1-loop IR divergence scale shown by the dashed black line.

coupling in the Banks-Zaks theory for $\epsilon=-1 / 10$ at 1 loop (diverging) and at 2 loops (converging to a fixed point).

It is immediately clear from the running of the coupling that the infrared dynamics, being conformal, is quite distinct from the chiral symmetry breaking QCD scenario. In particular, instead of becoming nonperturbative in the IR, it remains within the perturbative regime until it finally reaches a conformal theory in the deep infrared. We consider the epsilon regime in which the 2-loop analysis remains trustworthy. It would be interesting to extend the present work to higher loops [20,46,47]. Due to the perturbative control, we can fully include the fermion effects at 1-loop order by including their contribution to the beta function of the gauge coupling.

It is particularly interesting to investigate the massdeformed perturbative conformal field theory as argued first in [48]. Starting with fermions, all of the same mass $m \ll \Lambda_{c}$, the running is given in the top panel of Fig. 5. In the deep UV, at energies higher than the fermion mass $m$, the running is dominated by the free fixed point. At energies below the fermion mass, fermions can be integrated out. In the perturbative regime of the conformal window, we can follow the perturbative flow down to $m$. At energies lower than the common fermion mass, one enters the YM regime. In a mass-independent scheme (although our results for physical quantities are scheme independent) one matches the pure YM coupling with the one with massless fermions at the scale $m$, and the YM running takes over as shown in the bottom panel of Fig. 5.

In QCD one needs to take particular care of the lowenergy fermion modes when the hard common fermion mass is sufficiently small. This is the case since these modes are delocalized and feel the presence of the instanton

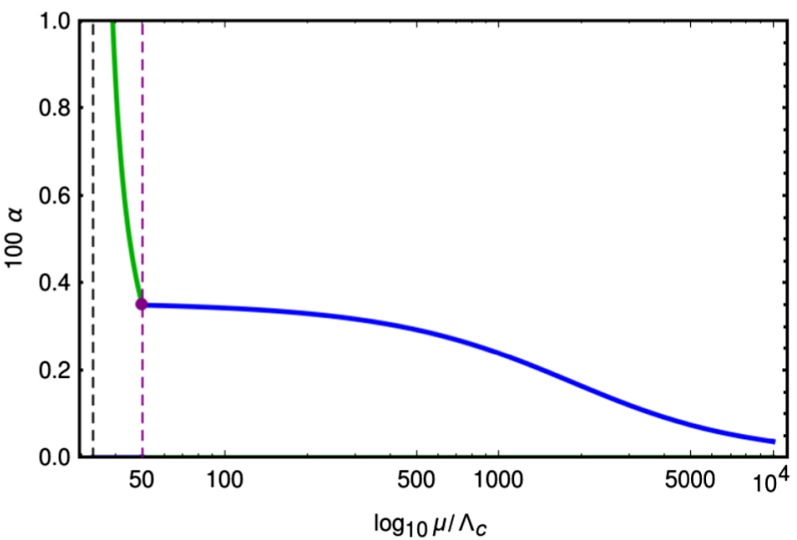

FIG. 5. The blue line shows the Banks-Zaks running for $\epsilon=-1 / 10$, and the green line corresponds to the pure YM running. The purple dot shows the matching couplings at the fermion mass scale, which is given by the dashed purple line. The dashed black line is the scale $\Lambda_{\mathrm{YM}}$, and the scale $\mu=\Lambda_{c}=1$ cannot be shown due to the use of log scale on the horizontal axis.

medium. In the perturbative regime of the conformal window, one can continue lowering the fermion mass all the way to zero because the coupling is guaranteed to stay perturbative down to the fermion mass scale. Above the common fermion mass energy, no condensate can form because the theory can be made arbitrarily weakly interacting [40]. As the fermions become massless, we expect the instantons to melt away and the vacuum-to-vacuum transition amplitude due to instantons to vanish.

To take into account the full perturbative running, we consider the RG-improved master equation. The often-used naive 2-loop running approach (19) is valid only in the deep UV since it does not account for the Banks-Zaks IR fixed point. Let us thus look into the exact 2-loop RG running more closely.

We begin by defining the 't Hooft coupling,

$$
\alpha=\frac{g^{2} N_{c}}{(4 \pi)^{2}} .
$$

The 2-loop beta function of the gauge coupling in the presence of fermions can be written as

$$
\mu \partial_{\mu} \alpha \equiv \beta_{\alpha}=-B \alpha^{2}+C \alpha^{3} .
$$

Here, $B=-4 / 3 \epsilon$ and $C=25+26 / 3 \epsilon$, and the physical control parameter is given by

$$
\epsilon=\frac{N_{f}}{N_{c}}-\frac{11}{2}<0 .
$$

The exact 2-loop running is given by [6]

$$
\alpha(\mu)=\frac{\alpha_{*}}{1+W(z(\mu))},
$$


where

$$
\alpha_{*}=B / C
$$

is the IR Banks-Zaks fixed point, and $W$ stands for the Lambert (or product log) function. Note that $z(\mu)$ will be defined shortly. Expansion around $\mu \rightarrow \infty$ yields Eq. (19). The RG running given by (45) is manifestly bounded, and it interpolates between the Gaussian fixed point $\alpha(\mu \rightarrow \infty)=0$ for infinite energies (a.k.a. asymptotic freedom) and an interacting fixed point $\alpha(\mu \rightarrow 0)=\alpha_{*}$ in the IR (a.k.a. conformal field theory), as can be seen from Fig. 4.

Just as confining QCD has a natural mass scale $\Lambda_{\mathrm{QCD}}$, there is an equivalent natural mass scale in the conformal window of QCD. The natural mass scale, referred to as $\Lambda_{c}$, is the 2-loop RG-invariant scale (just as $\Lambda_{\mathrm{QCD}}$ is the 1-loop RG-invariant scale). This means that $\mu \partial_{\mu}\left(\Lambda_{c}\right)$ vanishes to linear order. One can arrive at the expression for $\Lambda_{c}$ as follows. The gradient of the beta function $\partial_{\alpha} \beta_{\alpha}(\alpha)$ vanishes for $\alpha=2 / 3 \alpha_{*} \equiv \alpha_{c}$. The scale at which one reaches this value of the coupling is critical in the sense that, at this scale, the gauge coupling changes scaling from a canonical one to a non-Gaussian one. Solving for this energy scale, we get

$$
\mu\left(\alpha_{c}\right) \equiv \Lambda_{c}=\left(2 e^{-\frac{1}{2}}\right)^{-1 / \theta_{*}}\left(1-\frac{\alpha}{\alpha_{*}}\right)^{-1 / \theta_{*}} \mu,
$$

which is precisely the 2-loop RG-invariant scale $\Lambda_{c}$. In the definition, $\theta_{*}$ is the eigenvalue of the RG flow at the nonGaussian fixed point,

$$
\theta_{*}=\left.\frac{\partial \beta_{\alpha}}{\partial \alpha}\right|_{\alpha_{*}}=\alpha_{*} B
$$

It is worth emphasizing that what vanishes at the scale $\Lambda_{c}$ is only the gradient of the beta function, and not the beta function itself. This is crucial because zeros of the beta function are zeros of the flow of the couplings; thus they represent the fixed points of the flow. However, the zeros of the gradient of the beta function are points where the second derivative of the coupling with respect to the renormalization scale $\mu$ vanishes; i.e., the scale $\Lambda_{c}$ can be seen as an inflection point on the plots of the running coupling. See Figs. 4 and 5 for examples.

Inserting the RG improvements in the 1-loop master equation (16), we have

$$
\begin{aligned}
d_{2 L}(\rho)= & C_{c f} m^{N_{f}} \rho^{N_{f}-5}(b \log M \rho)^{2 N_{c}} e^{-\frac{8 \pi^{2}}{g_{2 L}^{2}}} \\
= & C_{c f} \exp (1 / 2-\log 2)^{-\frac{8 \pi^{2}}{g_{*}^{2}}} m^{N_{f}} \rho^{N_{f}-5} \\
& \times\left(\rho \Lambda_{c}\right)^{\frac{1}{2} B N_{c}}(b \log M \rho)^{2 N_{c}} W(z(\rho))^{\frac{8 \pi^{2}}{g_{*}^{2}}}
\end{aligned}
$$

In the above expression we use $C_{c f}$ as defined in (15), $\Lambda_{c}$ as defined in (47),

$$
z(\rho)=e^{1 / 2-\log 2}\left(\rho \Lambda_{c}\right)^{-\alpha_{*} B},
$$

the 1-loop beta coefficient $b$ given in (14), and the 1-loop RG-invariant scale $M$,

$$
M=\frac{1}{\rho_{c}} \exp \left(-\frac{1}{b} \frac{8 \pi^{2}}{g\left(\rho_{c}\right)^{2}}\right)=\Lambda_{c} \exp \left(-\frac{3}{2} \frac{C}{B^{2}}\right) .
$$

Setting $\rho^{2} \rightarrow \overline{\rho^{2}}$ in the second line of (50), the expression for the instanton density takes a form which is similar to what we had in the pure YM case. In fact, defining

$f(\bar{\rho})=C_{c f} \exp \left(\frac{1}{2}-\log 2\right)^{-\frac{8 \pi^{2}}{g_{*}^{2}}}\left(\frac{b}{2} \log M^{2} \overline{\rho^{2}}\right)^{2 N_{c}} W\left(\overline{\rho^{2}}\right)^{\frac{8 \pi^{2}}{g_{*}^{2}}}$,

we can write the 2-loop instanton density as

$$
d_{2 L}(\rho)=f(\bar{\rho}) m^{N_{f}} \rho^{N_{f}-5}\left(\rho \Lambda_{c}\right)^{\frac{1}{2} B N_{c}} .
$$

From here we find $\overline{\rho^{2}}$ analogously to the derivation of (33). The result can be put in the same form, with

$$
\nu=\frac{1}{2}\left(\frac{1}{2} B N_{c}+N_{f}-4\right) .
$$

The minimization of the partition function can now be performed in complete analogy to the derivation of the average instanton number in the pure YM theory, and we obtain

$$
\langle N\rangle=V \Lambda_{c}^{4}\left[\Gamma(\nu)\left(\frac{m}{\Lambda_{c}}\right)^{N_{f}} f(\bar{\rho})\left(\beta \gamma^{2} \nu\right)^{-\frac{\nu}{2}}\right]^{\frac{2}{2+\nu}} .
$$

Comparing to (34), the most notable difference is the appearance of the RG-invariant scale $\Lambda_{c}$ instead of the IR-divergence scale $\Lambda \simeq \Lambda_{\mathrm{YM}}$. Another important point is that $\tilde{\beta}^{2 N_{c}}$ is replaced by $\left(\frac{m}{\Lambda_{c}}\right)^{N_{f}} f(\bar{\rho})$, which renormalizes the 1-loop result (34). The partition function still has the same form (35) as in the pure YM case, but with new values for $\nu$ and $\langle N\rangle$.

Solving the equations for $\bar{\rho}$ and $N / V$ analogously to the pure YM case leads us to the results shown in Fig. 6. The results can be interpreted as follows. We solve for the instantons in the full Banks-Zaks theory, including both gauge fields and fermions, but it turns out that there are no instanton solutions above the fermion mass scale. Another logical possibility remains - that the instantons are truly infrared objects whose energy scale is below the fermion mass scale. In order to check this hypothesis, we have to solve the equations that are valid below the fermion mass 


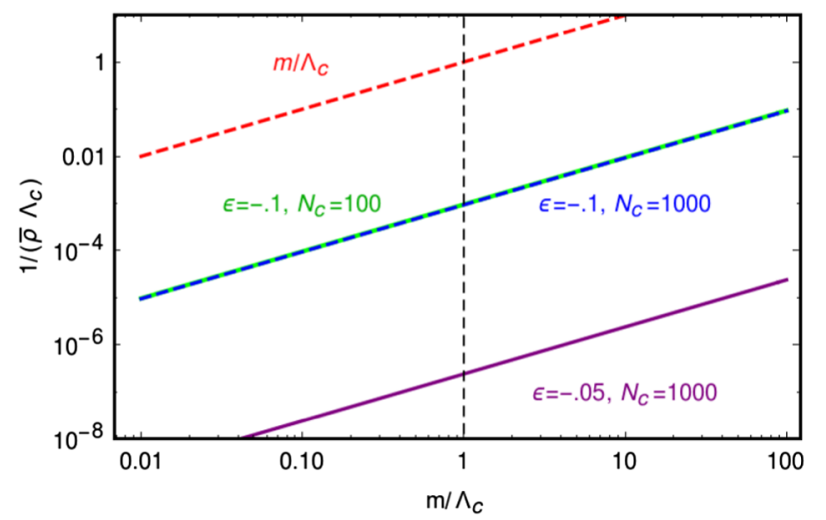

FIG. 6. The figure shows the inverse of $\bar{\rho}$ measured in units of $\Lambda_{c}$ for various choices of $\epsilon$ and $N_{c}$ (green, blue, and purple). For fixed $\epsilon=-0.1$, changing $N_{c}$ from 100 to 1000 changes $\bar{\rho}$ by less than $2 \%$. For $\epsilon=-0.1$ and $N_{c}=1000$, changing the parameter $a$ from 1 to 0.1 has less than a $0.1 \%$ effect. The dashed red line shows the fermion mass $m$ in units of $\Lambda_{c}$.

scale - namely, large- $N_{c}$ equations that we solved in the previous section. The above discussion leads us to look for the solution below the energy scale $m$, where running of the couplings is given by pure YM beta functions. ${ }^{4}$ This leads us to consider Eqs. (33) and (34) again.

We know from Sec. II B that the solutions for instantons in the pure YM theory are internally consistent, meaning that $\bar{\rho}^{-1} \gg \Lambda_{\mathrm{YM}}$. When solving the equations for the BZ theory, since we did not find any solutions for $\bar{\rho}^{-1}>m$, we additionally have to make sure that the consistency condition $\bar{\rho}^{-1}<m$ is met when using the pure YM running coupling. By ensuring that instantons live between the energy scales $\Lambda_{\mathrm{YM}}$ and $m$, we can guarantee that the full mass-deformed CFT is internally consistent.

Our results are shown in the top panel of Fig. 7, which shows the ratio of $m$ to $\bar{\rho}^{-1}$ as a function of $m$ measured in units of $\Lambda_{c}$. Results for $\bar{\rho}$ are well within the required consistency range, demonstrating that the theory possesses what could be described as large- $N_{c}$ pure-YM instantons. The bottom panel shows the inverse instanton length as a function of mass, and we can clearly see the power law decrease of the instanton scale as $m$ is taken to zero.

As an additional consistency check, one may study the behavior of $\Lambda_{\mathrm{YM}}$. In fact, here it is not an arbitrary number, but it is specified by the following 1-loop matching conditions:

$\frac{8 \pi^{2}}{g_{\mathrm{YM}}^{2}(m)} \equiv-\frac{11}{3} N_{c} \log \left(a \frac{\Lambda_{\mathrm{YM}}}{m}\right)=\frac{8 \pi^{2}}{g_{B Z}^{2}(m)}=\frac{N_{c}}{2 \alpha(m)}$,

\footnotetext{
${ }^{4}$ Equivalently, we may look for solutions using the running coupling defined as a piecewise function, equal to the BZ running coupling beyond energy $m$ and equal to matching pure YM running coupling below $m$.
}
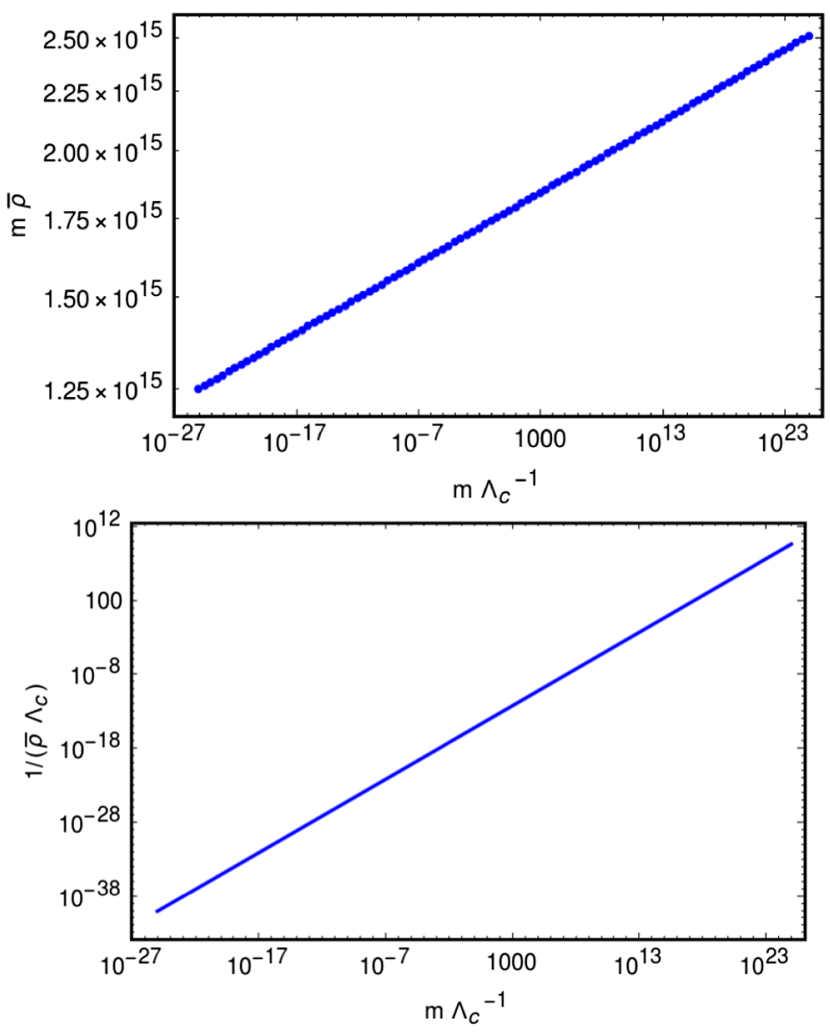

FIG. 7. We take $a=1 / 10, N_{c}=1000$, and $\epsilon=-1 / 10$. The top panel shows the instanton scale in the deep IR w.r.t. the fermion mass. The bottom panel shows $\bar{\rho}^{-1}$ as a function of $m$. Numerical values are predominantly determined by $\epsilon$, with a very mild dependence on $a$ and $N_{c}$.

which yields

$$
\Lambda_{\mathrm{YM}}=\frac{m}{a} \exp \left(-\frac{3}{22} \frac{1}{\alpha(m)}\right)
$$

For small enough $\epsilon$ the exponential term is flat as a function of $m$, so the dependence on mass here is essentially linear. Finally, we can measure $\bar{\rho}$ in units of $\Lambda_{\mathrm{YM}}$, and what we find is that it is flat as a function of $m$, taking the value $\bar{\rho}=0.390 \Lambda_{\mathrm{YM}}^{-1}$ for $a=1 / 10, N_{c}=1000$, and $\epsilon=-1 / 10$. See Fig. 8. Since the instantons in this theory are effectively large- $N_{c}$ pure-YM instantons, Fig. 8 effectively shows the same information as Fig. 1, with a minor numerical difference coming from using full 2-loop beta functions in the former case and the asymptotic expansion of the beta functions in the latter case.

Let us now discuss the instanton energy and the topological susceptibility. Since the couplings are renormalized at the energy scale corresponding to the inverse of the average instanton size, and since the instanton size turns out to be such that they sit well within the pure YM regime, the analysis closely follows the pure YM case. In particular, the partition function again takes the simple form (35), with $\langle N\rangle$ and $\nu$ given by (34) and (33), respectively. The total 


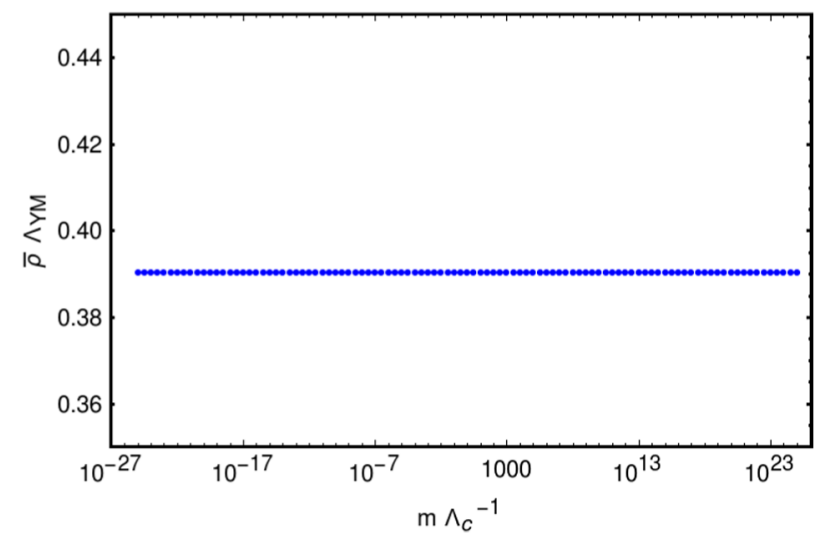

FIG. 8. The figure shows $\bar{\rho}$ in units of $\Lambda_{\mathrm{YM}}$ as a function of $m$. We fix $a=1 / 10, N_{c}=1000$, and $\epsilon=-1 / 10$.

energy is given by a sum of the free energy term, $\langle N\rangle$, and the interaction term. The interaction term comes from the derivative of the partition function w.r.t. $\beta=8 \pi^{2} / g_{2 L}^{2}(\bar{\rho})$. This dependency is hidden in $\langle N\rangle$ where it appears in the same form as it did in the pure YM case, which means the interaction energy can again be written as $\left\langle U_{\text {int }}\right\rangle=\nu\langle N\rangle /(2 \beta)$. The ratio of the interaction energy to the free energy thus follows the curve shown in Fig. 3. In fact, the shape of that curve changes significantly if 2-loop running is used instead of the 1-loop running, and the overall method is more stable when compared to the QCD case. If one fixes $m, N_{c}$, and $a$ (e.g., $m=1 / 10 \Lambda_{c}$, $N_{c}=1000, a=1 / 10$ ), one can study $\bar{\rho}$, in units of $\Lambda_{\mathrm{YM}}$, as a function of $\epsilon$ and find that it is constant (and in our example) equal to $\bar{\rho}=0.390 \Lambda_{\mathrm{YM}}^{-1}$. The reason why $\bar{\rho}(\epsilon)$ is constant in units of $\Lambda_{\mathrm{YM}}$ is related to the fact that $\Lambda_{\mathrm{YM}}$ decreases rapidly with decreasing $|\epsilon|$, thus compensating for the rapidly growing $\bar{\rho}$ in units of $\Lambda_{c}$.

The determination of the topological susceptibility proceeds as described in the previous section [see Eq. (41)]. As we discussed above, $\Lambda_{\mathrm{YM}} \bar{\rho}$ is essentially $m$ and $\epsilon$ independent (see Fig. 8). In this sense $N / V$ depends only on the explicit factor $\bar{\rho}^{-4}$. It is then clear that $N / V$ will rapidly decrease with decreasing $m / \Lambda_{c}$ when measured in units of $\Lambda_{c}^{-4}$, but it will be constant if measured in units of $\Lambda_{\mathrm{YM}}^{-4}$. This is confirmed in Fig. 9.

\section{SAFE INSTANTONS}

Here, we extend the instanton calculus to asymptotically safe quantum field theories starting with the first discovered controllable asymptotically safe four-dimensional gauge theory, here dubbed LISA [5].

\section{A. Controllable instantons in UV-safe gauge-Yukawa theories}

LISA consists of an $S U\left(N_{c}\right)$ gauge field coupled to $N_{f}$ vectorlike fermions and a scalar field. Besides the gauge coupling, there are Yukawa couplings and two scalar
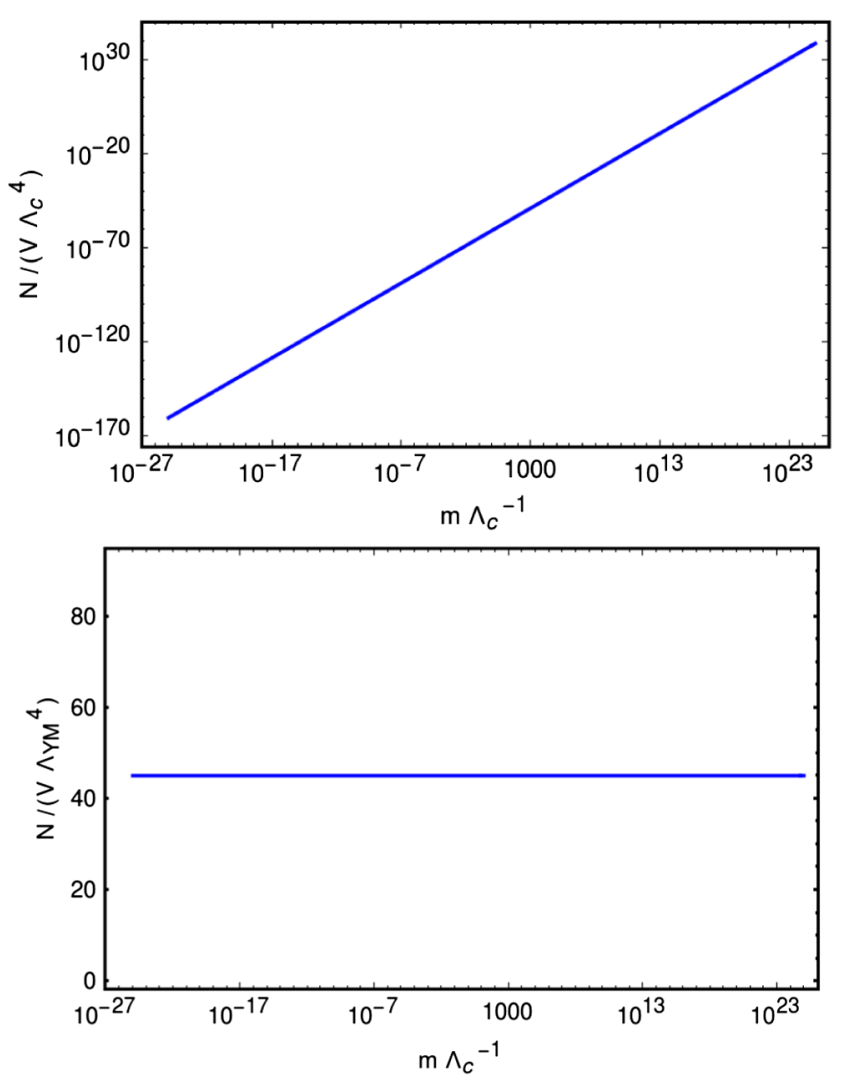

FIG. 9. The top panel shows the density of the instantons per unit volume measured in units of $\Lambda_{c}^{-1}$. The bottom panel shows the same quantity measured in units of $\Lambda_{\mathrm{YM}}^{-1}$. The exact value of $N / V$ depends on the fudge factor $a$. The bottom panel shows $N /\left(V \Lambda_{\mathrm{YM}}^{4}\right)=99.268$ obtained for $a=1 / 10$. Decreasing $a$ by $1 / 2$ increases $N / V$ by $30 \%$, and decreasing $a$ further by a factor of $1 / 2$ decreases $N / V$ by an additional $23 \%$.

self-couplings. At 2-loop order the beta function of the gauge coupling has the LO term exactly as in (43), but the cubic term becomes

$$
\left[\left(25+\frac{26}{3} \epsilon\right) \alpha-2\left(\frac{11}{2}+\epsilon\right)^{2} \alpha_{y}\right] \alpha^{2},
$$

where $\alpha_{y}=y^{2} N_{c} /(4 \pi)^{2}$ and $y$ is the Yukawa coupling. In the Veneziano limit, the theory admits a perturbative interacting UV fixed point. At the fixed point, the value of the gauge coupling, the Yukawa coupling, and scalar self-couplings all have values of order $\epsilon$, where $\epsilon$ is again given by (44), but this time it is positive because asymptotic freedom is lost. To simplify the discussion, we treat the Yukawa interaction in an effective manner according to which we substitute the fixed point value of the Yukawa coupling, $\alpha_{y}^{*}$, in the above expression. ${ }^{5}$ for the cubic term,

\footnotetext{
${ }^{5}$ This procedure, justifiable in perturbation theory, preserves, as it should, the safe behavior of the running gauge coupling interpolating between a Gaussian FP in the IR and a perturbative, non-Gaussain FP in the UV.
} 
leading exactly to the beta function (43), with $B=-4 \epsilon / 3$ and

$$
C=-\frac{2}{3} \frac{57-46 \epsilon-8 \epsilon^{2}}{13+\epsilon}
$$

Both $B$ and $C$ being negative, the fixed point appears at the physical value $\alpha_{*}=B / C>0$. Note that this would not be possible without the inclusion of scalars in the theory since the contribution of the Yukawa coupling was necessary for flipping the sign of the cubic term.

From here on, it is clear that running of the gauge coupling in LISA (in this slightly simplified form) is analogous to that in the Banks-Zaks theory, with UV and IR reversed, although the dynamics is profoundly different in nature.

In particular, the 2-loop running gauge coupling is again given by (45). The difference, of course, is in the fact that the argument of the Lambert function $z(\rho)$ that is given in (51) now runs inversely proportional to $\rho$ because $B=$ $-4 \epsilon / 3$ changes sign with respect to the IR interacting fixed point.

We include the fermion effects following the previous section. The running coupling behaves according to Fig. 10. Because the theory is noninteracting in the IR, there are no condensates of any kind forming at the fermion mass scale, and thus zero modes are managed in the same straightforward manner as in the previous section (see [36]). One major difference of LISA with respect to the Banks-Zaks theory, discussed in the previous section, is the presence of the Yukawa interactions. Let us therefore reiterate that, since the theory is in the controllable, perturbative regime at all energy scales above the fermion mass scale, no condensate forms, be it scalar, fermion, or gauge. Additionally, we keep the information about the Yukawa interactions, as discussed above, by considering its

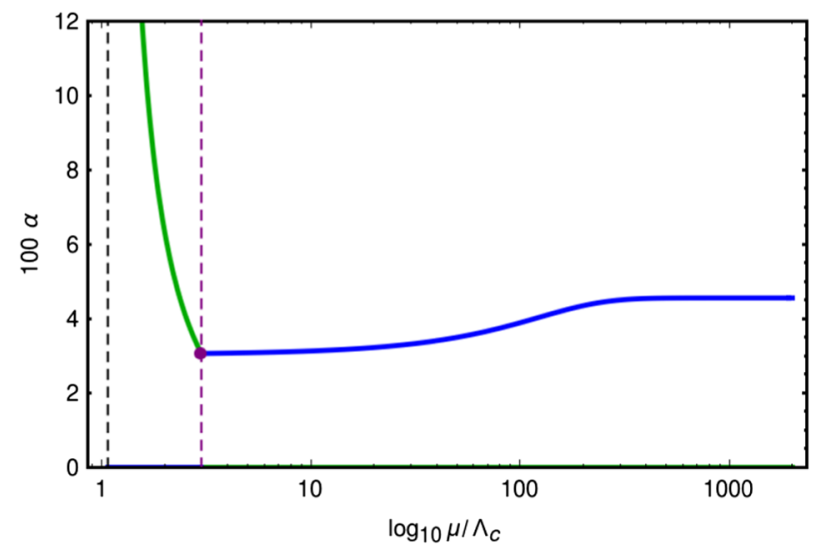

FIG. 10. The blue line shows the LISA running for $\epsilon=1 / 10$, and the green line corresponds to the pure YM running. The purple dot shows the matching couplings at the fermion mass scale, which is given by the dashed purple line. The black dashed line is the scale $\Lambda_{\mathrm{YM}}$. effects on the running of the gauge beta function. For these reasons, we can reasonably expect that there are no additional zero modes to consider, and that in our treatment the full effect of the Yukawa interactions has been captured by the modified gauge RG flow.

Computation of the average instanton size and the density of instantons per unit volume proceeds analogously to the perturbative interacting IR fixed point theory presented in the previous section. In particular, the 2-loop instanton density is given by (50). The main difference is that the argument of the Lambert function, given in (51), now grows with $\rho$ due to $B=-4 / 3 \epsilon<0$. In the remaining terms of (50) with explicit power-law dependence on $\rho, N_{f}$ dominates over $1 / 2 B N_{c}$, so the fact that $B$ changes sign here is irrelevant.

From this 2-loop density, one can obtain the effective 2-loop density $\mu(\rho)$ in a similar way as done in the IR interacting case. It will therefore again lead to the expression (32). Note that $\beta$ is just shorthand for $8 \pi^{2} / g^{2}$, so it does not change sign w.r.t. the IR interacting theory; in fact, we still have a Gaussian suppression of the IR instantons. The expectation value of $\rho$ that we obtain is given in (33), with $\nu$ given by (55). Here, $\beta$ is still positive, so $\nu$ has to be positive too if $\bar{\rho}$ is to be positive. In fact, $1 / 2 B N_{c}=-4 / 6 \epsilon N_{c}$, whereas $N_{f}=(11 / 2+\epsilon) N_{c} \simeq 11 / 2 N_{c}$; thus, $\nu$ is clearly positive in both the BZ and LISA theories.

Finally, $\langle N\rangle$ takes the same form (56) as in the BZ case, with $\nu, \beta$, and $f(\rho)$ appropriately modified. Solving the equations for $\langle N\rangle$ and $\bar{\rho}$ numerically, we find results similar to the BZ instantons. In particular, using the LISA beta functions, we find solutions for $\bar{\rho}$, with the instanton scale still smaller than the fermion mass, which means that the results are not consistent (see Fig. 11). We then solve the equations using the pure YM beta functions and find solutions with the instanton scale in the window between the IR $\Lambda_{\text {YM }}$ scale (which is expected from Sec. II B) and the fermion decoupling scale (which is a nontrivial consistency check). For the results in LISA, see Fig. 12.

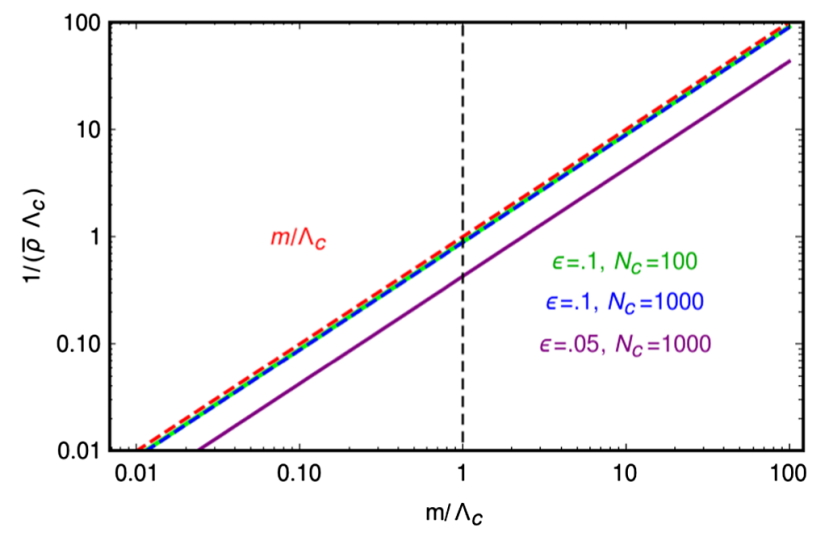

FIG. 11. The figure shows solutions for $\bar{\rho}$, obtained using LISA beta functions, for various choices of $\epsilon$ and $N_{c}$ (green, blue, and purple). The dashed red line shows the fermion mass. 

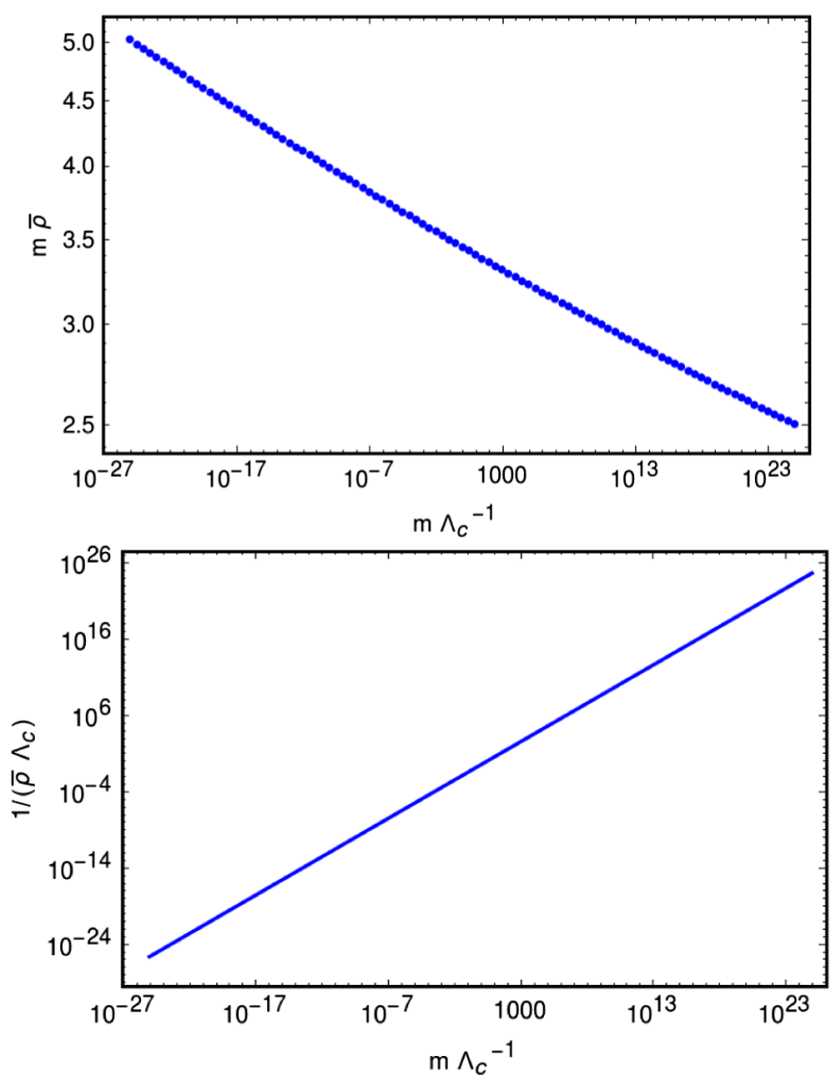

FIG. 12. The top panel shows the ratio $m / \bar{\rho}^{-1}$, and the bottom panel shows $\bar{\rho}^{-1}$ as functions of $m$. In both figures, $a=1 / 10$, $N_{c}=1000$, and $\epsilon=1 / 10$.

It is of some interest to compare Figs. 12 and 7. One interesting feature is that, for the chosen parameters, the instanton scale is about 1 order of magnitude smaller than the fermion mass in the case of LISA, but it is more than 16 orders of magnitude smaller than the fermion mass in the BZ theory. Here, the difference arises because in the infrared LISA is free rather than interacting. Further, in both cases the instanton scale $\bar{\rho}^{-1}$ always has to lie below the mass scale $m$, which explains the fact that the lines in the bottom panels have the same tendency to grow with $m$. Finally, from the top panels we see that the ratio $m / \bar{\rho}^{-1}$ grows with $m$ in BZ theory but decreases in LISA. In fact, the BZ theory is noninteracting in the UV, and the higher the energies at which we decouple fermions, the higher the IR instanton scale seems to be. This pattern is found in the LISA case as well. This behavior is related to how $\beta=8 \pi^{2} / g^{2}$ enters Eqs. (34) and (33).

We can study $\bar{\rho}$ in units of $\Lambda_{\mathrm{YM}}$ as a function of $m$, but it is clear that the results are described by Fig. 8; i.e., in units of $\Lambda_{\mathrm{YM}}$ the solution reproduces the BZ result. The same holds for $\langle N\rangle / V$ in units of $\Lambda_{\mathrm{YM}}^{-4}$, which is shown in the bottom panel of Fig. 9. For the instanton density in units of $\Lambda_{c}^{-4}$, see Fig. 13.

Because of the perturbative nature of the UV interacting fixed point, we have been able to extend the instanton

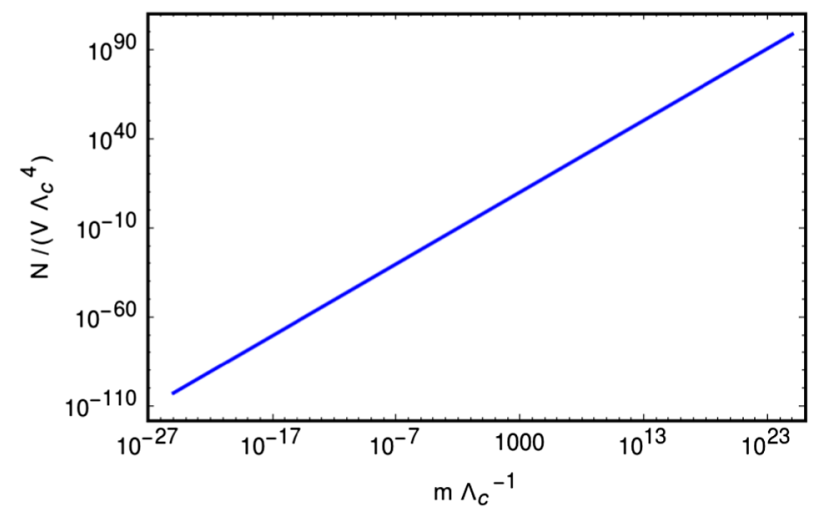

FIG. 13. The figure shows the density of the instantons per unit volume measured in units of $\Lambda_{c}^{-4}$.

calculus to controllable, asymptotically safe, quantum field theories.

\section{B. Safe QCD instantons: The large- $N_{f}$ story}

In LISA theory [5] elementary scalars and their induced Yukawa interactions crucially help tame the ultraviolet behavior of the overall gauge-Yukawa theory. Scalars, however, are not needed at a finite number of colors and a very large number of flavors for non-Abelian gaugefermion theories, as reviewed and further analyzed in [22].

Consider an $S U\left(N_{c}\right)$ gauge theory with $N_{f}$ fermions transforming according to a given representation of the gauge group. We consider the theory for a number of flavors above which asymptotic freedom is lost, i.e., $N_{f}^{A F}>11 C_{G} /\left(4 T_{R}\right)$, where the first coefficient of the beta function changes sign. Although we do not need to specify the fermion representation, we consider here the fundamental representation for which the relevant group theory coefficients are $C_{G}=N_{c}, \quad C_{R}=\left(N_{c}^{2}-1\right) / 2 N_{c} \quad$ and $T_{R}=1 / 2$. At 1-loop order the theory is simultaneously free in the infrared (non-Abelian QED) and trivial, meaning that the only way to take the continuum limit (i.e., sending the Landau pole induced cutoff to infinity) is for the theory to become noninteracting. At 2 loops Caswell [49] demonstrated that an UV interacting fixed point (asymptotic safety) cannot arise near the loss of asymptotic freedom, implying that safety can only occur above a certain critical number of flavors. This possibility has been (re)investigated in [22] at large $N_{f}$ and a fixed number of colors, for which the beta function is given by [17-20]

$$
\beta(A)=\frac{2 A}{3}\left(1+\sum_{i=1}^{\infty} \frac{H_{i}(A)}{N_{f}^{i}}\right),
$$

where we define the following large- $N_{f}$ normalized coupling: 


$$
A=\frac{N_{f}}{8 \pi^{2}} g^{2} .
$$

The functions $H_{i}(A)$ result by resumming an infinite set of Feynman diagrams at fixed order $i$ in the large $-N_{f}$ expansion $[17,18]$. Most importantly, to first order in large $N_{f}$, there is already a fixed point,

$$
A^{*}=3-e^{\left(-8 \frac{N_{f}}{N_{c}}+18.49-5.26 \frac{N_{c}^{2}-1}{2 N_{c}^{2}}\right)} .
$$

We now attempt to approximate the overall behavior of the beta function in order to estimate the instanton properties for this theory. Let us therefore write the 1-loop running as

$$
\beta_{1 L} \equiv \frac{8 \pi^{2}}{g^{2}}=-b \log \left(a_{L P} \Lambda_{L P} \rho\right)
$$

and the 2-loop running as

$$
\beta_{2 L}=\beta_{1 L}+\frac{b^{\prime}}{b} \log \beta_{1 L},
$$

where the 1-loop and 2-loop coefficients are given in (14) and (19). We fix the fudge factor $a_{L P}$ by requiring that the 2-loop running $g^{2}$ matches the UV FP value of $g^{2}$ at the 1-loop divergent scale $\Lambda_{L P}$.

Let us consider the specific examples $N_{f}=100, N_{c}=3$ for which $a_{L P}=1.189$. The running couplings are given in Fig. 14. There are three energy windows: The lowest one is below the fermion mass scale, the intermediate one runs up to the 1-loop divergent scale $\Lambda_{L P}$ (Landau pole), and the highest one is above the scale $\Lambda_{L P}$. In the highest energy window, we do not consider the RG running, but instead

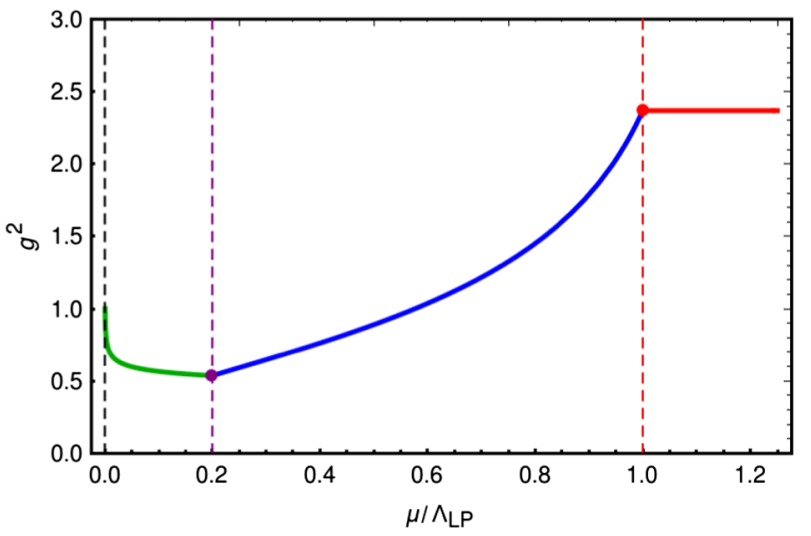

FIG. 14. Flow of the coupling in the mass-deformed large- $N_{f}$ UV conformal window. Pure Yang-Mills 1-loop running is shown in green. Matching to 2-loop QCD running, shown in blue, is at the fermion mass scale, given by the dashed purple line. We match 2-loop QCD running to the UV FP value, shown in red, at the 1-loop UV divergence scale $\Lambda_{L P}$, shown by the dashed red line.

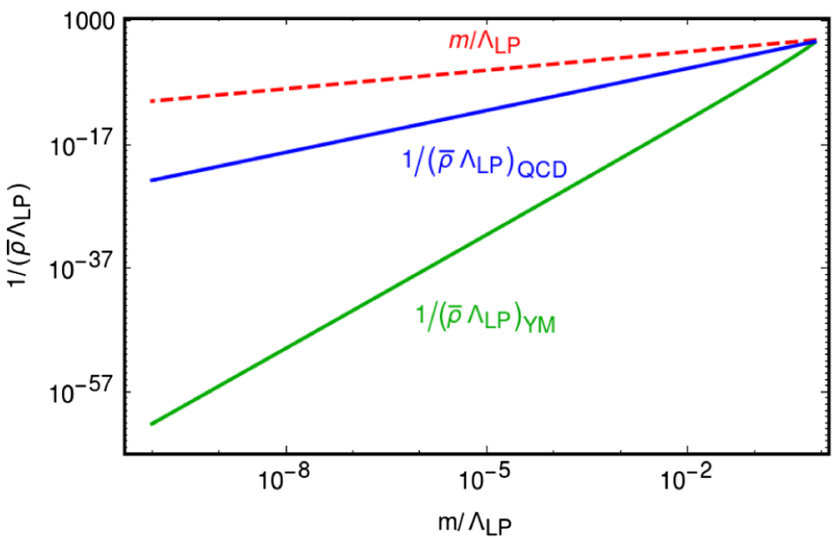

FIG. 15. The dashed red line shows the fermion mass scale. Solving for the $1 / \bar{\rho}$ using 2-loop QCD beta functions, we find the solutions shown in blue. This puts instantons below the fermion decoupling scale, which makes the solutions inconsistent. Next, we solve for the $1 / \bar{\rho}$ using 2-loop pure YM beta functions, and we find the solutions shown in green. We see that the instanton scale is still below the fermion mass scale and is thus consistent.

keep the coupling constant since it reaches the fixed point value at $\Lambda_{L P}$.

We only consider fermion masses $m<\Lambda_{L P}$, and in Fig. 15, we plot the results for the inverse instanton size (in units of $\Lambda_{L P}$ ) in the lower two energy windows. We see that using the naive running couplings, the instanton scale turns out to be very small, similarly to what we found in BZ and LISA. This is perhaps not surprising given the fact that we adopt a naive setup that makes use of a rough 2-loop approximation.

\section{CONCLUSIONS}

We investigated the instanton dynamics for fundamental field theories featuring either an asymptotically safe or free dynamics. In order to make the work self-contained, we provided a brief review of the role of instantons for YM and QCD dynamics, including the limitations of the instanton calculus. Within the asymptotically free scenario, we ventured into the perturbative regime of the QCD conformal window. Here, we determined, by extending the calculus to 2 loops, the number of instantons per unit volume as a function of a common fermion mass.

We then extended the instanton calculus to the case of controllable asymptotically safe theories. Here, the nontrivial UV dynamics demands the immediate use of higher order results. As for the conformal window case, we determined the fermion mass dependence of the instanton density. We further discussed the finite number of colors and large number of flavors limit.

It is interesting to observe that in all considered cases, the instantons appear as a fully IR phenomenon. In fact, in each theory we find instantons only below the fermion (common) mass scale. To the loop order that we are working, below the fermion mass scale there are only 
gauge fields present, so the instantons that we find are effectively large- $N_{c}$ pure Yang-Mills instantons. These are well known to be internally consistent, so we additionally verify that these solutions are consistent in the context of the UV complete theories that they belong to.

In the future one will extend the instanton calculus in order to cover a wider range of number of flavors within the calculable regime of UV and IR conformal windows. The ambitious goal is to determine to which extent the instanton dynamics is responsible for the loss of conformality once the number of flavors drops below a certain critical value for which either UV or IR conformality is lost in the respective safe or free conformal windows.

\section{ACKNOWLEDGMENTS}

The work of F. S. is partially supported by the Danish National Research Foundation under Grant No. DNRF:90.
[1] K. G. Wilson, Phys. Rev. B 4, 3174 (1971).

[2] K. G. Wilson, Phys. Rev. B 4, 3184 (1971).

[3] D. J. Gross and F. Wilczek, Phys. Rev. D 8, 3633 (1973).

[4] H. D. Politzer, Phys. Rev. Lett. 30, 1346 (1973).

[5] D. F. Litim and F. Sannino, J. High Energy Phys. 12 (2014) 178.

[6] D. F. Litim, M. Mojaza, and F. Sannino, J. High Energy Phys. 01 (2016) 081.

[7] F. Sannino and I. M. Shoemaker, Phys. Rev. D 92, 043518 (2015).

[8] S. Abel and F. Sannino, Phys. Rev. D 96, 056028 (2017).

[9] S. Abel and F. Sannino, Phys. Rev. D 96, 055021 (2017).

[10] G. M. Pelaggi, F. Sannino, A. Strumia, and E. Vigiani, Front. Phys. 5, 49 (2017).

[11] R. Mann, J. Meffe, F. Sannino, T. Steele, Z.-W. Wang, and C. Zhang, Phys. Rev. Lett. 119, 261802 (2017).

[12] G. M. Pelaggi, A. D. Plascencia, A. Salvio, F. Sannino, J. Smirnov, and A. Strumia, arXiv:1708.00437.

[13] A. D. Bond, G. Hiller, K. Kowalska, and D. F. Litim, J. High Energy Phys. 08 (2017) 004.

[14] T. Banks and A. Zaks, Nucl. Phys. B196, 189 (1982).

[15] F. Sannino, Acta Phys. Pol. B 40, 3533 (2009).

[16] C. Pica, Proc. Sci., LATTICE2016 (2016) 015.

[17] A. Palanques-Mestre and P. Pascual, Commun. Math. Phys. 95, 277 (1984).

[18] J. A. Gracey, Phys. Lett. B 373, 178 (1996).

[19] B. Holdom, Phys. Lett. B 694, 74 (2010).

[20] C. Pica and F. Sannino, Phys. Rev. D 83, 035013 (2011).

[21] R. Shrock, Phys. Rev. D 89, 045019 (2014).

[22] O. Antipin and F. Sannino, Phys. Rev. D 97, 116007 (2018).

[23] K. A. Intriligator and B. Wecht, Nucl. Phys. B667, 183 (2003).

[24] D. M. Hofman and J. Maldacena, J. High Energy Phys. 05 (2008) 012.

[25] K. Intriligator and F. Sannino, J. High Energy Phys. 11 (2015) 023.

[26] S. P. Martin and J. D. Wells, Phys. Rev. D 64, 036010 (2001).

[27] N. Seiberg, Nucl. Phys. B435, 129 (1995).
[28] B. Bajc and F. Sannino, J. High Energy Phys. 12 (2016) 141.

[29] B. Bajc, N. A. Dondi, and F. Sannino, J. High Energy Phys. 03 (2018) 005.

[30] M. Shifman, Advanced Topics in Quantum Field Theory (Cambridge University Press, Cambridge, England, 2012).

[31] E. V. Shuryak, World Sci. Lect. Notes Phys. 71, 1 (2004); 8, 1 (1988).

[32] S. Coleman, Aspects of Symmetry: Selected Erice Lectures (Cambridge University Press, Cambridge, England, 1988).

[33] T. Schäfer and E. V. Shuryak, Rev. Mod. Phys. 70, 323 (1998).

[34] A. I. Vainshtein, V. I. Zakharov, V. A. Novikov, and M. A. Shifman, Usp. Fiz. Nauk 136, 553 (1982) [Sov. Phys. Usp. 25, 195 (1982)].

[35] A. A. Belavin, A. M. Polyakov, A. S. Schwartz, and Yu. S. Tyupkin, Phys. Lett. 59B, 85 (1975).

[36] G. 't Hooft, Phys. Rev. D 14, 3432 (1976); 18, 2199(E) (1978).

[37] C. W. Bernard, N. H. Christ, A. H. Guth, and E. J. Weinberg, Phys. Rev. D 16, 2967 (1977).

[38] C. W. Bernard, Phys. Rev. D 19, 3013 (1979).

[39] M. A. Shifman, A. I. Vainshtein, and V. I. Zakharov, Nucl. Phys. B163, 46 (1980).

[40] D. Diakonov and V. Yu. Petrov, Nucl. Phys. B272, 457 (1986).

[41] C. G. Callan, Jr., R. F. Dashen, and D. J. Gross, Phys. Rev. D 17, 2717 (1978).

[42] E. V. Shuryak, Nucl. Phys. B203, 116 (1982).

[43] D. Diakonov and V. Yu. Petrov, Nucl. Phys. B245, 259 (1984).

[44] W. E. Caswell and F. Wilczek, Phys. Lett. 49B, 291 (1974).

[45] T. Schäfer, Phys. Rev. D 66, 076009 (2002).

[46] T. A. Ryttov and R. Shrock, Phys. Rev. D 83, 056011 (2011).

[47] T. A. Ryttov and R. Shrock, Phys. Rev. D 94, 105015 (2016).

[48] F. Sannino, Phys. Rev. D 82, 081701 (2010).

[49] W. E. Caswell, Phys. Rev. Lett. 33, 244 (1974). 